\title{
Robustness of the Warm Arctic/Cold Eurasian Signature within a Large Ensemble Model Experiment
}

\author{
Masatake E. Hori ${ }^{1}$ and Kazuhiro Oshima ${ }^{2}$ \\ ${ }^{1}$ Japan Agency for Marine-Earth Science and Technology/Institute of Arctic Climate and Environment Research \\ (JAMSTEC/IACE), Yokosuka, Japan \\ ${ }^{2}$ Institute for Environmental Sciences, Rokkasho, Aomori, Japan
}

\begin{abstract}
We use two groups of 100-member ensemble AGCM experiment to investigate the robustness and probabilistic nature of the Warm Arctic/Cold Eurasian (WACE) pattern with or without strong warming SST trend and sea-ice reduction. Model ensembles successfully simulate a distribution of trend coefficients close to that of observation. Results show that the recent trend in WACE pattern is driven by the warming of the Arctic SST, but the pattern itself is not amplified between the warming and non-warming experiment and cannot explain the current cooling trend of the mid-latitudes. We argue that the difference in sea-ice condition regulates the more extreme cases of the pattern thereby contributing to the positive trend in WACE pattern similar to that of observation.

(Citation: Hori, M. E., and K. Oshima, 2018: Robustness of the warm arctic/cold Eurasian signature within a large ensemble model experiment. SOLA, 14, 69-73, doi:10.2151/sola.2018-012.)
\end{abstract}

\section{Introduction}

Presently, there is a hot debate on the topic of whether the rapid warming in the Arctic and the associated change in sea-ice can trigger a cold anomaly in the mid-latitudes (Overland 2016; Fransis 2017).

Inoue et al. (2012) was one of the first papers to point out this particular anomaly where the warming Arctic and change in seaice concentration over the Barents Sea influence the dominant cyclone migration pathway creating an extension of the Siberian High along the coast of the Eurasian continent leading to a coldair outburst in the mid-latitudes.

However, the discussion remains whether this anomaly is indeed induced by the rapidly declining sea-ice concentration or an artifact of natural variability. Mori et al. (2014) showed through 100 -member ensemble simulation of an atmospheric general circulation model (AGCM) that the second empirical orthogonal function (EOF) pattern reflecting such Arctic warming and mid-latitude cooling is robust and is approximately independent of the Arctic Oscillation (AO) signature. The pattern is named Warm Arctic/Cold Eurasian (WACE) and will be used to designate the pattern throughout this study.

Crasemann et al. (2017) also used an AGCM setup and found that under an altered sea-ice condition, blocking over the Scandinavian Peninsula is pronounced during December and January and a negative North Atlantic Oscillation (NAO) signature becomes more frequent during February and March, creating an overall effect of WACE.

On the other hand, there are studies such as McCusker et al. (2016) which discuss the possibility that such anomaly arises from the natural variability in the Barents Sea region and do not require a change in sea-ice concentration. Collow et al. (2018) shows through both AGCM and Coupled (ocean-atmosphere) General Circulation Model (CGCM) runs, that Sea Surface Temperature (SST) changes in the Arctic creates a mean warming response

Corresponding author: Masatake E. Hori, 2-15 Natsushima-cho, Yokosuka 237-0061, Japan. E-mail: mehori@jamstec.go.jp. over Eurasia and while individual model ensemble with a cooling response do exist, it is within the range of internal variability. Screen (2017) also points out that while negative NAO like pattern emerges from sea-ice changes, it does not lead to change in the mid-latitudes due to the offsetting by advection of warmer air masses.

Overland et al. (2016) summarize that due to the shortage in observational data and limitation in modeling performance in long-range forecasting, one cannot assert at this state the prevalence of robust external forcing in the Arctic over the more chaotic internal variability of the atmosphere.

In this study, we attempt to overcome this limitation by utilizing an AGCM experiment framework designed to probabilistically address the change in SST and sea-ice under the current historical configuration against a non-warming configuration where the trend in SST is removed.

Comparing the non-warming experiment to the historical model setup, we try to approach the recent trend observed in the WACE pattern in a probabilistic manner, quantifying the distribution of linear trend coefficients and comparing it to the real-world observation. Also, we compare the amplitude of the WACE pattern between a model setup of strong positive Sea Surface Temperature (SST) trend in the Arctic and the lack thereof. Finally, the sensitivity of the WACE pattern against sea-ice is investigated.

\section{Data and study method}

As a reference, we use the surface temperature from the JRA55 reanalysis dataset made by the Japan Meteorological Agency (Kobayashi et al. 2015) which is in 1.25 latitude/longitude resolution from the year 1959-2017.

We use the "Database for Policy Decision-Making for Future Climate Change" (d4PDF) dataset which is an ensemble climate dataset designed for probabilistic diagnosis of historical climate variability and impact assessment (Mizuta et al. 2017). d4PDF consists of a historical and a natural forcing run on the MRIAGCM3.2, an AGCM with a horizontal resolution of $60 \mathrm{~km}$. In this study, we use the historical climate simulation (HPB) and the non-warming simulation (HPB-NAT) which extends from 1951-2010, and each consists of 100 members. The HPB-NAT experiment prescribes the SST, sea-ice concentration (AICE), and sea-ice thickness (SIT) based on COBE-SST2 (Hirahara et al. 2014) and perturbs the SST by $30 \%$ of the inter-annual standard deviation for each ensemble with sea-ice adjusted to be consistent with the given SST whereas the HPB-NAT removes the trend in SST and prescribes external forcing such as greenhouse gasses to preindustrial level.

December to February (DJF) average of surface temperature and Sea Level Pressure (SLP) is used throughout this study with the climatology defined as the average of the total dataset.

\section{Results}

One way to quantify the internal variability within multiple model ensembles is to assume that the ensemble mean represents the forced response and define the desired internal variability (in 
a)

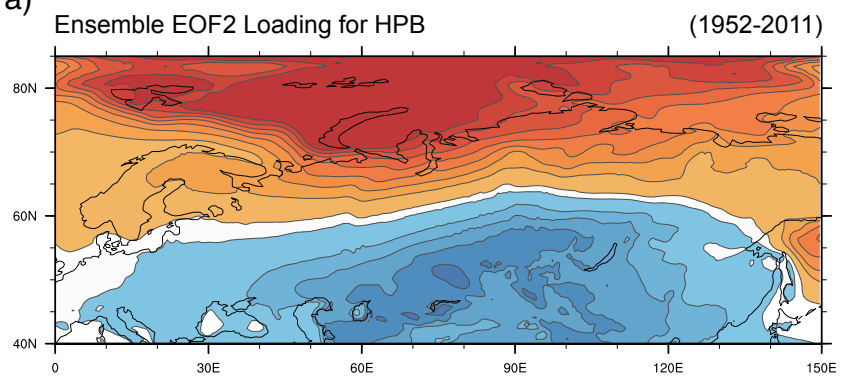

b)

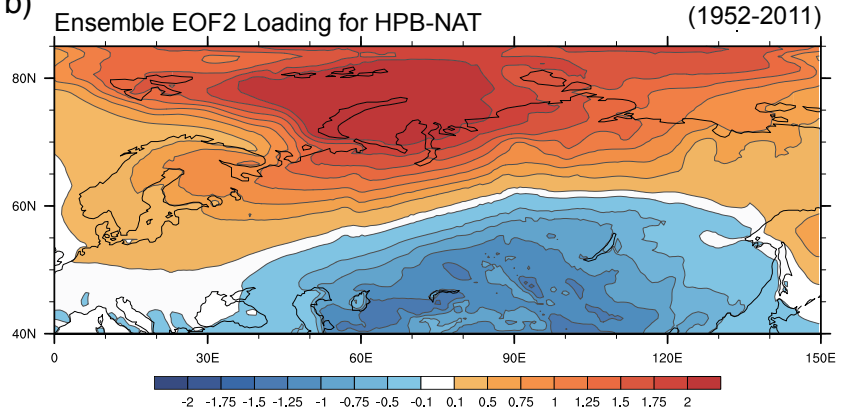

Fig. 1. Ensemble mean EOF2 loading pattern for the DJF averaged surface temperature in the region $0^{\circ} \mathrm{E}-150^{\circ} \mathrm{E}, 40^{\circ} \mathrm{N}-85^{\circ} \mathrm{N}$ for a) $\mathrm{HPB}$, and b) HPB-NAT. EOF is taken on each model ensemble for the year 1952-2011 using the anomaly from the climatological mean.

this case, WACE) as a departure from this ensemble mean. However, in this study we attempt to describe the manifestation of the WACE pattern in each ensemble under each specific SST forcing and collectively show the natural variability of the pattern within HPB and HPB-NAT. To do this, we first establish a reference EOF loading pattern based on the JRA-55 dataset. Region selected is $0^{\circ} \mathrm{E}-150^{\circ} \mathrm{E}, 40^{\circ} \mathrm{N}-90^{\circ} \mathrm{N}$, slightly different from the definition used in Mori et al. (2014). Next, for each ensemble run of both HPB and HPB-NAT experiments, we perform the same EOF analysis. We check each EOF loading pattern for the leading mode being the region-wide warming and the second mode being the WACE dipole pattern.

Figure 1 shows the ensemble averaged loading pattern for the second mode of EOF for a) HPB and b) HPB-NAT. The loading pattern is similar to that of Mori et al. (2014), with a distinct warming in the Barents Sea accompanied by cooling over the Eurasian continent, a signature feature of WACE. Comparing the two pattern, HPB shows a stronger loading in the Arctic Ocean than HPB-NAT and the mid-latitude cooling being more zonally distributed. This may be due to the fact that within the ensembles, there are variety of structures in the dipole pattern with the mid-latitude cooling centered more towards the eastern or western flank of the ensemble loading pattern.

To check if the EOF loading pattern is stable within the dataset, Fig. 2 shows the Taylor diagram (Taylor 2001) of EOF2 loading pattern against the reference EOF2 pattern of JRA-55. In the HPB experiment, the field correlation of the EOF2 loading pattern lies in the range of $0.68-0.94$ with a standard deviation ranging from $0.73-1.08$. Loading pattern for HPB-NAT experiment shows a weaker correlation in the range of $0.45-0.91$, with a weaker standard deviation of $0.63-1.03$. Overall, a lower correlation corresponds to the shift in center of action in the loading pattern with either the Arctic warming shifting eastwards towards the Kara Sea or the mid-latitude cooling displaced westward or eastward than the reference JRA-55 loading pattern. A stronger standard deviation corresponds to a stronger center of action in the Arctic and a weaker loading in the mid-latitudes, whereas a weaker standard deviation shows a more gradual north/south gradient in the dipole pattern with the emphasis on the mid-latitudes (see Fig. S1). For a)
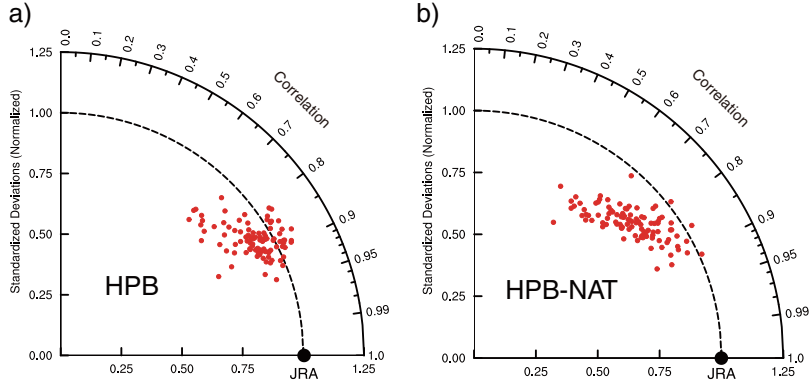

Fig. 2. Taylor diagram of EOF 2 loading pattern for each model ensemble within a) HPB experiment and b) HPB-NAT experiment. Black circle corresponds to the reference loading pattern of JRA-55. Radial distance is the ratio of normalized root-mean-square (RMS) difference, and the angle corresponds to the pattern correlation coefficient relative to JRA-55 EOF 2 loading pattern. a)

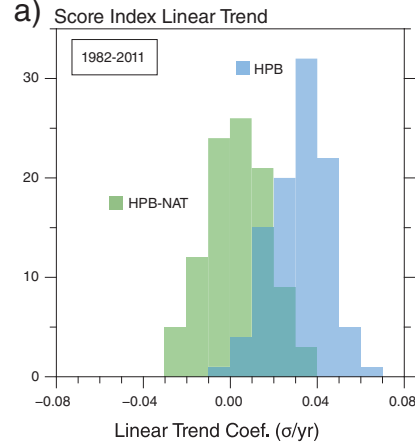

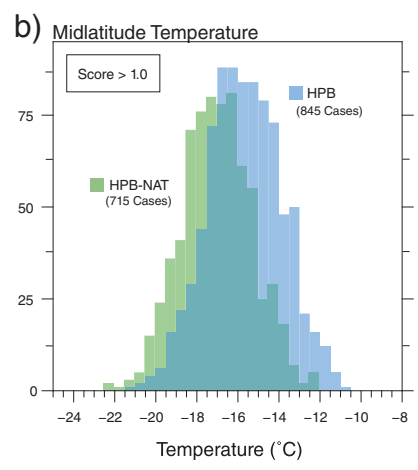

Fig. 3. a) Histogram of linear trend coefficient of EOF2 score index for the latter half of HPB and HPB-NAT experiment. b) histogram of mid-latitude surface temperature anomaly for the region $40^{\circ} \mathrm{E}-120^{\circ} \mathrm{E}, 45^{\circ} \mathrm{N}-65^{\circ} \mathrm{N}$ taken for all cases of normalized score index over 1.0. Blue shade corresponds to the HPB experiment and green shade for the HPB-NAT experiment.

both HPB and HPB-NAT the loading pattern is in one cluster, and the field correlation and amplitude bear a good resemblance to the observation so that we assume all EOFs successfully represents the WACE pattern with various manifestations within the ensembles.

Using the normalized score index for each ensemble, we take the linear trend coefficient for the latter half of the experiment, the year 1982-2011 where the warming trend in the Arctic and SeaIce change is more pronounced. Figure 3 a shows the histogram of trend coefficients for all ensembles. By experimental design, the SST in HPB-NAT is de-trended and the corresponding linear trend in the score index is thus centered at zero with a distribution due to perturbation between ensembles. Compared to HPB-NAT, the trend coefficient for HPB is mostly positive with an median value of $0.032 \sigma / y r$ and is well separated from HPB-NAT. The realworld trend in WACE pattern based on the same years of JRA-55 yields $0.040 \mathrm{\sigma} / \mathrm{yr}$ which is strikingly close to the HPB average. It is also surprising that the most recent 30-year (1988-2017) trend coefficient for JRA-55 reaches $0.08 \sigma / \mathrm{yr}$, which is even beyond the most extreme case of the HPB experiment which is $0.064 \mathrm{\sigma} / \mathrm{yr}$.

Figure $3 \mathrm{~b}$ shows the distribution of area-averaged surface temperature in the region of $40^{\circ} \mathrm{E}-120^{\circ} \mathrm{E}, 45^{\circ} \mathrm{N}-65^{\circ} \mathrm{N}$ under the extreme case of WACE pattern defined by the normalized score index exceeding 1.0. HPB experiment has 845 cases, and HPBNAT has 715 cases of such winter. Mid-latitude cooling under an extreme WACE case has a median value of $-15.6^{\circ} \mathrm{C}$ and a standard deviation of $1.8^{\circ} \mathrm{C}$ in the HPB experiment whereas the median is $-16.9^{\circ} \mathrm{C}$ and the standard deviation is $1.72^{\circ} \mathrm{C}$ in the 

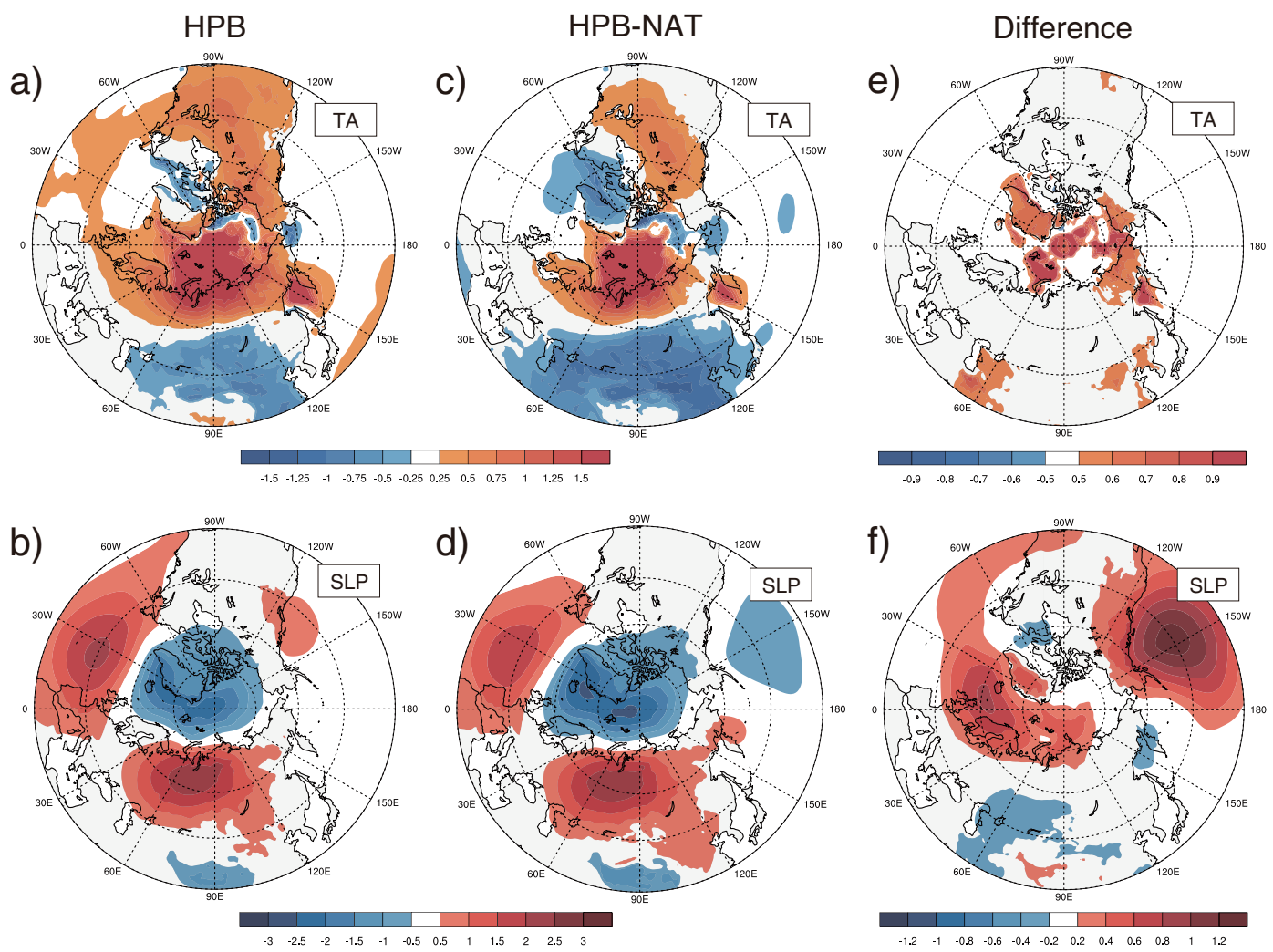

Fig. 4. Composite analysis of all cases shown in Fig. $3 b$ for a), c) surface temperature and b), d) SLP for HPB experiment, and HPB-NAT experiment respectively. Difference in ensemble mean for e) surface temperature and f) SLP.

HPB-NAT experiment. The difference in standard deviation is not significant, and a $1.32^{\circ} \mathrm{C}$ difference in median value can be attributable to the global warming trend within the model.

To examine the possibility that recent rapid warming in the Arctic is amplifying the WACE pattern, we perform a composite analysis of all cases in Fig. 3b. Figures $4 \mathrm{a}$ and $4 \mathrm{~b}$ shows the surface temperature and SLP composite for the HPB experiment, and Figs. $4 \mathrm{c}$ and $4 \mathrm{~d}$ that of the HPB-NAT experiment. Figures $4 \mathrm{e}$ and 4f shows the difference between the two experiments with HPBNAT subtracted from HPB. It should be noted that anomalies are relative to their respective climatology and the comparison signifies the difference in anomaly loading of the WACE pattern under HPB and HPB-NAT.

Figures $4 \mathrm{a}$ and $4 \mathrm{c}$ show a robust Arctic warming and a midlatitude cooling over the Eurasian continent centered around $50^{\circ} \mathrm{N}$ extending downstream towards East Asia. There is a modest warm anomaly over North America and cooling over Greenland which separates the pattern from an annular symmetric AO pattern. Mid-latitude cooling is more pronounced in the HPB-NAT experiment due to the fact that the region is significantly cooler in HPBNAT than in HPB in the latter half of the experiment used. Also, the difference in the north/south gradient of the EOF loading pattern as shown in Fig. 1 leads to more pronounced response in the Arctic for HPB and more response in the mid-latitudes for HPBNAT.

Figure $4 \mathrm{e}$ shows the difference in anomaly loading between HPB/HPB-NAT which is signified by a strong warming in the sea-ice boundary. It should be noted that due to the difference in climatology, the result is skewed towards the polar amplification pattern of HPB but not entirely identical (see Fig. S2).

This result shows that while WACE pattern is evident in both HPB and HPB-NAT experiments, recent warming in the Arctic does not amplify the structure of the pattern and warmer Arctic does not necessarily mean a colder mid-latitude.

Corresponding SLP pattern in Figs. $4 \mathrm{~b}$ and $4 \mathrm{~d}$ show a dis- tinctive NAO dipole pattern over the Atlantic and a positive SLP anomaly over the northern coastline of the Eurasian continent. The difference in SLP for HPB and HPB-NAT shows a weakening of the southern flank of the Icelandic low corresponding to a weaker NAO pattern and possibly a wavier jet-stream extending from the North Atlantic into the Barents Sea, similar to the atmospheric response to Arctic warming as discussed in Francis and Vavrus (2015).

If it is not the intensification of the WACE pattern itself under global warming that is driving the recent cooling in the Eurasian continent, the second possibility we must study is the increased probability of more extreme WACE pattern occurrences under the changing sea-ice boundary condition.

To illustrate this, we define the sea-ice interannual variability using the area averaged AICE data in the Barents Sea, namely the $0^{\circ} \mathrm{E}-80^{\circ} \mathrm{E}, 65^{\circ} \mathrm{N}-98^{\circ} \mathrm{N}$ region. Five years of large/small Barents Sea ice concentration in the latter half of the dataset is selected where 1989, 1998, 1999, 2003, 2004 model year is defined as the high-ice years, and years from 2006 to 2010 as the low-ice years. Perturbation of sea-ice in the ensembles is smaller than the inter-annual variability, therefore making it possible to compare the high-ice years to the low-ice years without normalization.

Figure 5a shows the EOF2 score index distribution for the high/low ice year which shows a shift towards positive and more extreme WACE cases in the low ice years. While this shows that the low sea-ice condition prefers a positive EOF2 corresponding to a stronger WACE pattern, it should be noted that this shift in distribution does not necessarily mean that a mid-latitude cooling is more preferred under the low sea-ice years. Due to the strong loading pattern in EOF2 over the Barents Sea, this shift in EOF2 score distribution can occur even when there is little or no cooling in the mid-latitudes.

To further elucidate this point, Fig. $5 \mathrm{~b}$ shows the ensemble mean composite difference of surface temperature for high/low sea-ice years with high sea-ice years subtracted from the low sea- 
a) EOF2 Score Distribution

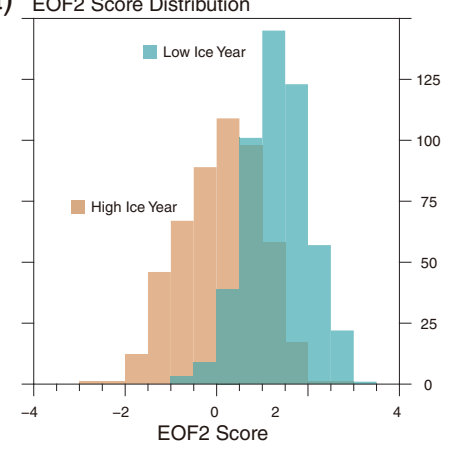

b)

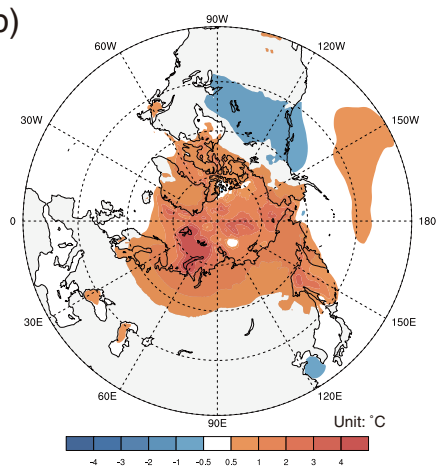

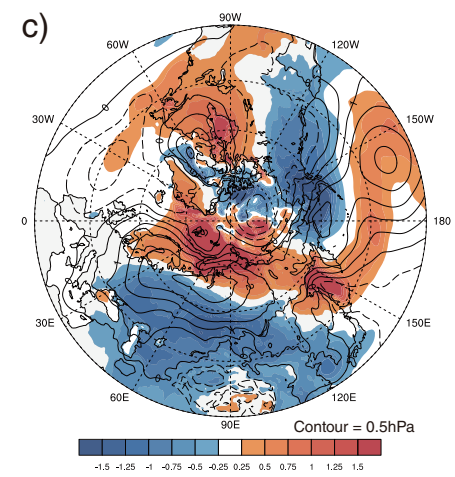

Fig. 5. a) Histogram of EOF 2 score taken for five high/low sea ice years in the Barents Sea, namely the $0^{\circ} \mathrm{E}-80^{\circ} \mathrm{E}, 65^{\circ} \mathrm{N}-98^{\circ} \mathrm{N}$ region. Red shade corresponds to the high ice year and blue shade to the low ice year, b) ensemble mean composite difference of surface temperature for high/low sea-ice years with high sea-ice years subtracted from the low sea-ice years, c) composite difference of 50 extreme cases of high/low ice years for the anomaly of surface temperature (shaded) and SLP (contour) for the HPB experiment.

ice years. Averaged over all members, the difference is mainly in the Arctic with no mid-latitude cooling.

However, there is possibility that the difference in sea-ice may lead to more extreme cases of the EOF2 loading pattern in the tail region of the distribution given in Fig. 5a. To elucidate this point, we examine 50 ensemble cases (out of 500 cases) with the strongest EOF2 score in each sub-sampled group of high/low seaice years to create a composite difference of the corresponding surface temperature (shades) and SLP (contour) in Fig. 5c. The result shows a warming in the Arctic Sea and cooling in the mid-latitudes zonally extending from $30^{\circ} \mathrm{E}-120^{\circ} \mathrm{E}$ and into East Asia and a positive SLP difference along the Northern coastline of the Eurasian continent which is the signature of a WACE pattern. Within the AGCM modeling framework of the d4PDF, it can be argued that the sea-ice boundary acts to change the probabilistic distribution of extreme cases of WACE to create a tendency of positive trend in the pattern.

\section{Conclusion and discussions}

In this study, we investigated the probabilistic distribution of the WACE pattern using the d4PDF dataset to infer the effect of recent Arctic warming on the structure and amplitude of the pattern and its dependence on sea-ice boundary condition. Using JRA-55 observation as a reference, we were able to extract a robust pattern of Arctic Warming and Eurasian Cooling both in the HPB historical experiment and the non-warming HPB-NAT experiment.

Comparing two experiments using the latter half of the dataset where Arctic warming is significant in the HPB experiment, we showed that the distribution of linear trend coefficient was positive in the HPB experiment whereas in the non-warming HPB-NAT it was collectively neutral with a nearly equal number of ensembles having a positive trend as much as the negative trend. Median value of the trend coefficients in HPB was $0.032 \mathrm{\sigma} / \mathrm{yr}$ which is very close to the real-world value of $0.04 \mathrm{\sigma} / \mathrm{yr}$.

The difference in the composite analysis of extreme cases of WACE pattern shows almost no difference in the mid-latitude cooling and a slightly warmer Arctic surface temperature in the HPB experiment suggesting that the structure of the WACE pattern does not change under strong polar amplification.

The possible source of positive trends in the WACE pattern in the HPB experiment is the sea-ice boundary in the Barents Sea where high/low ice concentration separated the EOF score into a positive leaning group and a more neutral group. A composite analysis of the more extreme cases within this group shows the distinctive north-south temperature dipole and intensification of the northern flank of the Siberian high thus creating more cold surges downstream (Inoue et al. 2012). However, taken as an ensemble mean, sea-ice do not systematically induce a mid-latitude cooling response as seen in Fig. 5b. The sensitivity of extreme cases in the WACE pattern should be further studied through comparisons against the $4^{\circ} \mathrm{K}$ global warming experiments.

It can be inferred that while the WACE pattern is a robust pattern of the northern hemisphere winter climate over the Arctic and the Eurasian continent, the structure itself is not a forced response under pronounced global warming, but instead changes its probabilistic occurrences through sea-ice boundary conditions in the Barents Sea.

Several limitations exist in this study with the most significant being that the d4PDF uses an AGCM and do not account for the feedback of the atmosphere nor the land-surface condition back towards the SST and sea-ice concentration.

Also, we have yet to describe the change in WACE pattern under the $4^{\circ} \mathrm{K}$ global warming experiment which is part of the d4PDF framework. This will be addressed in future study.

\section{Acknowledgments}

The authors are grateful to Dr. Kosuke Ito, editor of SOLA and two anonymous reviewers for their constructive comments. This study utilized the database for Policy Decision making for Future climate change (d4PDF), which was produced under the SOUSEI program.

Edited by: K. Ito

\section{Supplement}

Figure S1: Based on the Taylor diagram of Fig. 2, each figure shows the EOF2 loading pattern of a specific member in HPB with highest/lowest correlation and highest/lowest standard deviation relative to JRA-55. Figure S2: Ensemble mean difference in surface temperature climatology for HPB and HPB-NAT.

\section{References}

Collow, T. W., W. Wang, and A. Kumar, 2018: Simulations of Eurasian winter temperature trends in coupled and uncoupled CFSv2. Adv. Atmos. Sci., 35, 14-26, doi:10.1007/s00376017-6294-0.

Crasemann, B., D. Handorf, R. Jaiser, K. Dethloff, T. Nakamura, J. Ukita, and K. Yamazaki, 2017: Can preferred atmospheric circulation patterns over the North-Atlantic-Eurasian region be associated with arctic sea ice loss? Polar Sci., 14, 9-20, doi:10.1016/j.polar.2017.09.002.

Ebita, A., S. Kobayashi, Y. Ota, M. Moriya, R. Kumabe, K. Onogi, Y. Harada, S. Yasui, K. Miyaoka, K. Takahashi, H. Kama- 
hori, C. Kobayashi, H. Endo, M. Soma, Y. Oikawa, and T. Ishimizu, 2011: The Japanese 55-year Reanalysis "JRA-55": An interim report. SOLA, 7, 149-152, doi:10.2151/sola. 2011-038.

Francis, J. A., 2017: Why Are Arctic Linkages to Extreme Weather Still Up in the Air? Bull. Amer. Meteor. Soc., 98, 2551-2557, doi:10.1175/BAMS-D-17-0006.1.

Francis, J. A., and S. J. Vavrus, 2015: Evidence for a wavier jet stream in response to rapid Arctic warming. Environ. Res. Lett., 10, 014005, doi:10.1088/1748-9326/10/1/014005.

Handorf, D., K. Dethloff, S. Erxleben, R. Jaiser, and M. V. Kurgansky, 2017: Arctic-mid-latitude linkages in a nonlinear quasi-geostrophic atmospheric model. Adv. Meteor., 2017, doi: $10.1155 / 2017 / 2691368$

Hori, M. E., J. Inoue, T. Kikuchi, M. Honda, and Y. Tachibana, 2011: Recurrence of intraseasonal cold air outbreak during the 2009/2010 winter in Japan and its ties to the atmospheric condition over the Barents-Kara Sea. SOLA, 7, 2528, doi:10.2151/sola.2011-007.

Inoue, J., M. E. Hori, and K. Takaya, 2012: The role of Barents Sea ice in the wintertime cyclone track and emergence of a warm-Arctic cold-Siberian anomaly. J. Climate, 25, 25612568, doi:10.1175/JCLI-D-11-00449.1.

Kobayashi, S., Y. Ota, Y. Harada, A. Ebita, M. Moriya, H. Onoda, K. Onogi, H. Kamahori, C. Kobayashi, H. Endo, K. Miyaoka, and K. Takahashi, 2015: The JRA-55 reanalysis: General specifications and basic characteristics. J. Meteor. Soc. Japan, 93, 5-48, doi:10.2151/jmsj.2015-001.
McCusker, K. E., J. C. Fyfe, and M. Sigmond, 2016: Twenty-five winters of unexpected Eurasian cooling unlikely due to Arctic sea-ice loss. Nature Geosci., 9, 838-843, doi:10.1038/ NGEO2820.

Mizuta, R., A. Murata, M. Ishii, H. Shiogama, K. Hibino, N. Mori, O. Arakawa, Y. Imada, K. Yoshida, T. Aoyagi, H. Kawase, M. Mori, Y. Okada, T. Shimura, T. Nagatomo, M. Ikeda, H. Endo, M. Nosaka, M. Arai, C. Takahashi, K. Tanaka, T. Takemi, Y. Tachikawa, K. Temur, Y. Kamae, M. Watanabe, H. Sasaki, A. Kitoh, I. Takayabu, E. Nakakita, and M. Kimoto, 2017: Over 5,000 years of ensemble future climate simulations by $60-\mathrm{km}$ global and $20-\mathrm{km}$ regional atmospheric models. Bull. Amer. Meteor. Soc., 98, 1383-1398, doi:10.1175/BAMS-D-16-0099.1.

Mori, M., M. Watanabe, H. Shiogama, J. Inoue, and M. Kimoto, 2014: Robust Arctic sea-ice influence on the frequent Eurasian cold winters in past decades. Nature Geosci., 7, 869873, doi:10.1038/NGEO2277.

Overland, J. E., 2016: A difficult Arctic science issue: Midlatitude weather linkages. Polar Sci., 10, 210-216., doi:10.1016/ j.polar.2016.04.011.

Screen, J. A., 2017: The missing Northern European winter cooling response to Arctic sea ice loss. Nature Commun., 8, doi: $10.1038 /$ ncomms 14603 .

Taylor, K. E., 2001: Summarizing multiple aspects of model performance in a single diagram. J. Geophys. Res. Atmos., 106, $7183-7192$

Manuscript received 28 March 2018, accepted 22 May 2018

SOLA: https://www.jstage.jst.go.jp/browse/solal 